\title{
A vigilância alimentar e nutricional no Brasil
}

\section{Malaquias Batista Filho* Maria Anunciada F. Lucena** Maria Luiza M. Evangelista * *}

Com base em dois projetos em funcionamento (Recife, no Estado de Pernambuco, e Vale do Piancó, na Paraiba) descreve-se o Sistema de Vigilancia Alimentar e Nutricional do Brasil (SIVAN). Trata-se especificamente da organização institucional e operativa, coleta, processamento, análise e uso das informaçóes obtidas pelo SIVAN. Os dois projetos implantados são experimentais, nđo podendo, portanto, serem tomados como modelo já definidos. Os resultados obtidos são ainda preliminares e, por esta razão, não estão incluidos no presente documento. A nivel central, o Instituto Nacional de Alimentação e Nutrição (INAN) está reelaborando o projeto SIVAN sob novos conceitos, objetivos e diretrizes operacionais.

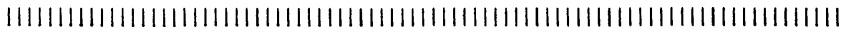

* Prof. Adjunto do Depto de Nu. trição, Recife. Prof. Visitante da Escola Nacional de Saúde Pública, FIOCRUZ, Rio de Janeiro.

**Prof. Adjunto do Dept? de Nutriçäo, Recife. Assessora do SISVAN Recife.

***Economista, coordenadora do IPLAN Paraiba.

\section{SITUAÇÃO ALIMENTAR E NUTRICIONAL}

Apesar de ser a 8 a economia do mundo, em termos de produto interno bruto (PIB), atingindo uma renda "per capita" de 2.200 dólares anuais, o modelo de desenvolvimento praticado no Brasil não possibilitou a correção de graves desajustes estruturais de seu processo econômico e social. São chocantes as estatísticas sobre o sistema pro. dução/distribuição de riquezas, sob os aspectos geográficos e sociais. A região Nordeste, por exemplo, reunindo 30\% da população do País, gera apenas $10 \%$ da renda nacional; sua população economicamente ativa, no meio rural, produz apenas 55 dólares mensais "per capita" classificandose entre as mais pobres do mundo ${ }^{6}$. No País como um todo, $8 \%$ das familias mais ricas consomem $62 \%$ dos bens e serviços disponíveis. Por outro lado, $9 \%$ da populaçá mais pobre participaria do mercado de forma residual, ou seja, $0,05 \%^{7}$.

Em razão desse quadro estrutural polarizado pelos extremos de riqueza e pobreza, o Brasil apresenta um perfil de saúde que reflete a coexistência de situações nosológicas opostas. Assim, entre as principais causas de morte agrupam-se as doenças cardiovasculares, infecciosas e parasitá-

Recebido para publicação em 08.10 .86 
rias, neoplasias e doenças do aparelho respiratório ${ }^{5}$. As mortes por diarréia e doenças respiratórias agudas, com freqüên. cia associadas à desnutrição, têm um peso muito expressivo no quadro de obituário ${ }^{15}$.

Não existem dados atualizados sobre a situação alimen. tar do País. Segundo o Banco Mundial, 67\% da população brasileira estaria com uma dieta restritiva em calorias, senso que, no Nordeste urbano, 91,5\% das pessoas não alcançariam as recomendações em energia.

\section{TABELA 1}

Estimativas da população com "déficit" energético no Brasil (1974-1975)

\begin{tabular}{|c|c|c|c|c|}
\hline \multirow{2}{*}{ Especificações } & \multicolumn{2}{|l|}{ Com "déficit" } & "Déficit & \multirow[t]{2}{*}{400 calorias } \\
\hline & $\begin{array}{c}\text { Em } 1.000 \\
\text { pessoas }\end{array}$ & & $\begin{array}{c}\text { Em } 1.000 \\
\text { pessoas }\end{array}$ & \\
\hline \multicolumn{5}{|l|}{ BRASIL } \\
\hline Urbano & 49027,9 & 76,5 & 14057,1 & 21,9 \\
\hline Rural & 23003,7 & 53,4 & 4514,0 & 10,5 \\
\hline Total & 72031,6 & 67,2 & 18571,1 & 17,3 \\
\hline \multicolumn{5}{|c|}{ NORDESTE DO BRASIL } \\
\hline Urbano & 13074,1 & 91,5 & 6952,6 & 48,7 \\
\hline Rural & 12378,6 & 69,8 & 2428,9 & 13,7 \\
\hline Total & 25425,7 & 79,5 & 9381,5 & 29,3 \\
\hline
\end{tabular}

Fonte: BIRD-Brasil: Human Resources Special Report. 1979.

Estima-se que menos de $5 \%$ da população apresenta um consumo deficitário de proteínas, o que levaria à conclusão de que, sob o ponto de vista calórico-preotéico,a restrição de energia seja o fator essencialmente limitante da alimen. $\operatorname{tação~}^{13}$.

Com exceção das zonas metropolitanas do Rio de Janeiro e Salvador, em todas as áreas amostrais estudadas o consumo médio de vitamina A não atingia os valores recomen. dados, encontrando-se sistematicamente nas familias rurais os níveis de adequação mais baixos ${ }^{16}$. No Nordeste rural, um estudo indicou que 77 das familias consumiam dietas com adequação de vitamina a baixo de $50 \%{ }^{10}$. As médias globais de ingestão de ferro são satisfatórias em quase todas as áreas estudadas. No entanto, quando se considera as famílias de baixa renda - mais de $65 \%$ da população brasileira tornam-se freqüentes os casos de inadequação. Presume-se, ademais, que o ferro alimentar seja de baixa biodisponibilidade.

São poucos os estudos que definem tipologias de famílias, segundo critérios sócioeconômicos para fins de pes. quisas sobre alimentação. Trabalhos efetuados a partir de 
categorias de despesas familiares (variável "proxi" de renda), a nível nacional, ou a partir do acesso à terra, no Nordeste rural, indicam que o valor da dieta se situa bem abaixo dos requerimentos nutricionais estabelecidos nas familias de estratos sociais inferiores ${ }^{1,19 *}$. Ressalta-se, nestes trabalhos, a deficiência da dieta em termos de energia, vitamina $\mathrm{A}$, ferro, riboflavina e niacina.

Um problema importante a ser considerado é o desmame precoce. Em 1980, 14,6\% das crianças nascidas nas cidades e $11,6 \%$ dos recém-nascidos nas áreas rurais jamais receberam leite materno. A média de desmamc completo estava abaixo de 3 meses 8 .

Segundo a classificação de Gomez ${ }^{9}$, cerca de $48 \%$ das crianças menores de 5 anos seriam portadores de algum grau de desnutrição. E $94 \%$ dos casos de desnutrição avançada (IIl grau) ocorreriam em menores de 2 anos, sendo esta uma característica epidemiológica importante do problema da desnutrição energético-protéica no Brasil (Tabela 2) *.

TABELA 2
*BATISTA FILHO, M. Nutrição, alimentação e agricultura no Nor. deste brasileiro. Trabalho apresentado no Seminário Internacional sobre Agricultura e Nutrição, Car. pina, Pernambuco, 1985, a ser publicado.
* VALENTE, J.C. Apud. BA. TISTA FILHO, M. \& BARBOSA, N.P. ${ }^{4}$

\section{Situação Nutricional de Crianças Brasileiras, segundo a adequação peso/idade}

\begin{tabular}{ccccc}
\hline \multirow{2}{*}{ Idades } & Normals & \multicolumn{3}{c}{ Desnutridos } \\
\cline { 3 - 5 } & & I grau & II grau & III grau \\
\hline Menores de 6 meses & 71,3 & 17,4 & 8,9 & 2,5 \\
$6-12$ meses & 65,2 & 22,3 & 11,0 & 1,4 \\
$1-2$ anos & 56,1 & 32,2 & 11,1 & 0,5 \\
$2-5$ anos & 47,0 & 38,1 & 14,8 & 0,2
\end{tabular}

lionte: VALINTE, J.C. Comunicação pessoal. INAN, Brasília, 1984.

Na zona rural do Nordeste, $71 \%$ das crianças são desnutridas, segundo a adequação peso/idade, sendo $22 \%$ na condição de II e III graus. A mediana de crescimento ponderal na categoria sócio-econômica de mais baixa renda (categoria de Despesa 4) se situava abaixo do percentil 3 do NCHS e $28 \%$ das crianças estavam abaixo do valor -2 S.D. da população de referência* ${ }^{*}$.

* BATISTA FILHO, op. cit.

Estima-se que $80 \%$ das formas moderadas e graves de des. nutrição ocorreriam em familias com menos de 2 salários mínimos, admitindo-se, por esta razão, esse nível de renda como discriminante da clientela a ser assistida pelos programas de alimentação e nutrição, em caráter prioritário ${ }^{2}$.

Não existem evidências de que o baixo peso ao nascer represente um problema relevante de saúde no Brasil. Sua prevalência seria de $8.5 \%{ }^{12}$. 
Embora ainda pouco estudada, considera-se que a anemia ferropriva constitui, em termos de prevalência, o se-

*VALENTE, C. \& GARCIA A.A.F. Apud BATISTA FILHO, $M .^{3}$ gundo maior problema de nutrição do Brasil. A tabela 3 é bem ilustrada, a este respeito ${ }^{19}$.

TABELA 3

Distribuição de anemias no Brasil (1982-1984)

\begin{tabular}{llrc}
\hline \multicolumn{1}{c}{ Estado/Localidade } & \multicolumn{1}{c}{ Grupos } & $\begin{array}{c}\text { Amostra } \\
\text { nọ }\end{array}$ & Prevalência \\
\hline São Paulo (várias localidades) & Gestantes & 1.616 & 31,6 \\
São Paulo (várias localidades) & $10-24$ meses & 508 & $66,0-79.0$ \\
São Paulo (várias localidades) & Homem (5-12 anos) & 1.277 & $43,8-57,0$ \\
Pará (Belém) & Gestantes & 762 & 65,1 \\
Pará (Belém) & 6 anos (H. M.) & 692 & 68,6 e 71,0 \\
Pará (Belém) & 6-14 anos (H. e M.) & 529 & 53,3 e 47,0 \\
Pará (Belém) & Mulheres adultas & 1.447 & 41,1 \\
Pernambuco (litoral) & Gestantes & 317 & 42,0 \\
Pernambuco (Recife) & Pop. em geral & 1.130 & 50,9 \\
Pernambuco (Mata) & Pop. em geral & 2.293 & 53,2 \\
Pernambuco (Agreste) & Pop. em geral & 1.736 & 32,7 \\
Paraíba (várias localidades) & Gestantes & 528 & $9,9-25,8$ \\
Paraíba (várias localidades) & 1 a 5 anos & 1.593 & $15,3-19,7$ \\
Paraiba (várias localidades) & Escolares & 761 & $15,0-19,2$
\end{tabular}

Fonte: INAN, Brasília, 1984 (Doc. Téc. 495)

A hipovitaminose A ainda não tem o seu quadro nosográfico bem estabelecido no Brasil. Estudos efetuados no Nordeste (Pernambuco e Paraíba) Norte (Pará) Sudeste (Minas Gerais e São Paulo) são indicativos de que o problema assume relevância de saúde coletiva, sendo frequente entre menores de 5 anos a ocorréncia de níveis de retinol sérico abaixo de $20 \mathrm{mcg} / 100 \mathrm{dl}$. (4,5). No semi-árido do Nordeste brasileiro $\left(940.000 \mathrm{~km}^{2}\right.$ e cerca de 12 milhões de habitantes) têm sido assinaladas freqüências elevadas de sinais oculares de hipovitaminose $\mathrm{A}^{14}$.

O bócio, em 1975, teria uma prevalência de 14,5\% em escolares $^{11}$.

\section{VIGILÂNCIA ALIMENTAR E NUTRICIONAL}

Antecedentes gerais - A criação do Instituto Nacional de Alimientação e Nutrição (1972) e a implementação do II Programa Nacional de Alimentação e Nutrição (1974) estabelecem, de fato, os marcos institucionais para a formalização de um compromisso político e administrativo com os problemas pertinentes a esta área.

No curso de reuniões técnicas do INAN e de discussoes em congressos e seminários nacionais e internacionais efeCadernos de Saúde Pública, R.J., 2 (3): 349-358, jul/set, 1986. 
tuados no Brasil, bem como em razão do incentivo de orga nizaçoes como a FAO e a OPAS, se considerou a importância e a conveniência de estabelecer atividades de vigilancia alimentar e nutricional no Brasil. Efetivamente, só a partir de 1983, o INAN passou a tomar medidas concretas neste sentido, sob a responsabilidade da sua Secretaria de Programas Especiais.

O Brasil tem, atualmente, cerca de 35 milhões de beneficiários de programas de alimentação e nutrição, nao contando, no entanto, com um sistema de informaçoes e análise que possa avaliar a pertinência das açóes propostas e seus resultados.

\section{OBJETIVOS}

- Atualizar o diagnóstico da situação nutricional, com atenção para os bolsões de pobreza, acompanhar sua avaliação e acionar dispositivos de alerta e intervenção em situações de grave risco nutricional.

- Promover e sistematizar articulações entre instituiçoes de pesquisas e órgãos executores envolvidos na problemática nutricional da população em seus diversos aspectos.

- Formular metodologias de operacionalização do SISVAN sobre fundamentaçбes estabelecidas pelo Instituto Nacional de Alimentação e Nutrição.

- Consolidar um banco de dados sobre alimentação e nutrição.

\section{DESCRIÇÃO DO SISVAN}

Existem de fato, duas experiências em andamento, nos Estados de Pernambuco e Pararba, ds quais se aplica esta descrição. Dois outros projetos devem ser implementados no Rio de Janeiro (FIOCRUZ) e São Paulo. Estão sendo analisadas as experiências em curso, abrindo-se a perspectiva de que os modelos em operação sejam profundamente modificados em seus aspectos conceituais, metodológicos, institucionais e administrativos.

- Instituição Responsável - Instituto Nacional de Alimentação e Nutrição (Ministério da Saúde), encarregado da coordenação, a nível nacional.

- Organização

No Estado de Pernambuco, o SISVAN CONTA COM A PARTICIPAÇÃO DA Secretaria de Saúde e do Instituto Nacional de Assistência Médica da Previdência Social (INAMPS), responsáveis pela prestação de serviços de saúde à popułação; Secretarias de Agricaltura e Educação e Fun- 
dação de Assistência ao Educando. O SISVAN/Recife é administrado pela Fundação Joaquim Nabuco (Ministério da Cultura), contando ainda com a assistência técnica do Departamento de Nutrição da Universidade Federal de Pernambuco.

Na Paraíba, o SISVAN é administrado através de convênio com o Instituto de Planejamento (IPLAN), do Governo do Estado. Participam também as Secretarias de Saúde do Estado, o Departamento de Nutrição da Universidade da Paraiba, Fundação Instituto Brasileiro de Geografia e Estatística, Empresa de Assistência Técnica e Extensão Rural e Secretarias de Agricultura e de Educação.

O gráfico a seguir define a composição do sistema sem implicar em subordinação hierárquica.

\section{COBERTURA}

No Estado de Pernambuco, o sistema está implantado em 5 unidades de saúde que atenderam 33\% da população-alvo. Na Pararba, e municípios do Vale do Piancó seriam assistidos pelos serviços de saúde. No Rio de Janeiro, a UTGSF presta assistência a $90 \%$ das mães e crianças menores de 1 ano residentes na área operativa (25.000 habitantes).

A coleta de dados antropométricos e os inquéritos de consumo alimentar (semestrais) incluem, no entanto, apenas amostras probabilisticas que permitam inferencias sobre as tendências destas variáveis.

Os dados de natureza sócioeconômica (custo dos alimentos, índice geral de preços ao consumidor, taxa de desemprego, produção agrícola consolidada, estimativa de safras e outras, são tomados de fontes primárias (locais) ou secundárias (estaduais e regionais), tendo, assim, coberturas geográficas e populacionais diferentes.

\section{ESTRUTURA OPERATIVA}

A nivel local a) serviços de saúde da Previdência (INAMPS) e dos Estados; b) serviços de extensao agrícola; c) escolas (na realidade ainda não integradas ao sistema); d) Instituiçбes de pesquisa (Fundação Joaquim Nabuco, FIPLAN, departamentos de nutrição das Universidades da Paraıba e Pernambuco). Estas unidades sao responsáveis pela coleta de informações a nivel de comunidade e pela prestação de serviços.

A nivel regional: Secretarias de Saúde, Educação, Comissão de Estudos e Planejamento Agrícola, EMATER, Fundação Joaquim Nabuco, FIPLAN, encarregados da supervisão, controle, processamento e análise de dados, elaboração e difusão de relatórios e recomendaçôes técnicas. 
$A$ nivel central: Instituto Nacional de Alimentação e Nutrição, responsável pela coordenação geral, decisões normativas, programáticas, políticas e apoio financeiro.

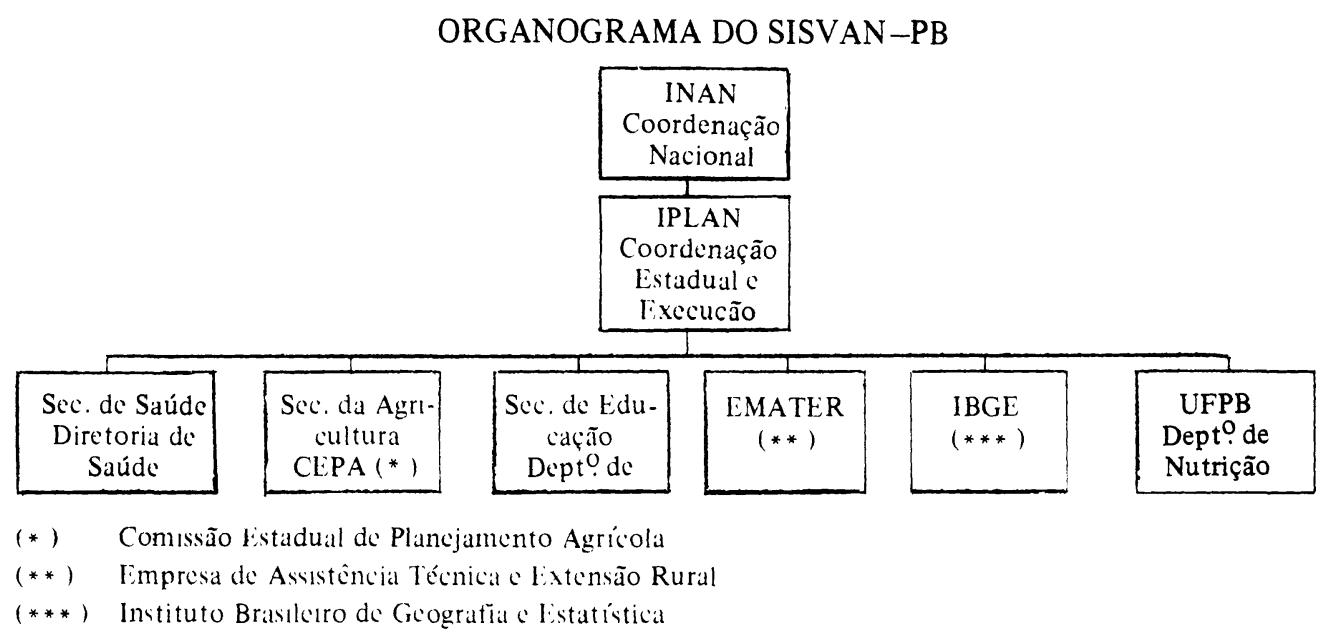

\section{FONTES DE DADOS}

Os dados antropométricos (peso, altura) são gerados pelos diferentes serviços de saúde que integram a rede. Não foi possível ainda sistematizar a coleta de dados clínicos e bioqufmicos (Vitamina A, hemoglobina) em grupos de alto risco.

Informações sobre preços de alimentos que formam a cesta básica são tomadas mensalmente nas cidades de Itapo. ranga (Paraıb) e Recife (PE), por pessoas contratadas pelo SISVAN. O consumo de alimentos a nível familiar é obtido através de inquérito recordatório de 24 horas, além de pesa. gem direta dos produtos.

As escolas produzem dados sobre matrícula, absenteismo e evasão escolar, não tomando ainda informações sobre estado de nutrição. As Comissões Estaduais de Planejamento Agrícola (CEPA) dispõem de serviços de coletas de dados sobre área plantada, estimativas de safras, etc.

Outras informações que alimentam o SISVAN são de fontes secundárias: taxa de desemprego, índice de preços ao consumidor, orçamentos familiares, dados demográficos e demais variáveis.

\section{FLUXO DAS INFORMAÇOES}

Os dados primários (peso, altura, preços de alimentos) gerados a nível local, são transcritos em formulários próprios e encaminhados a cada més ao SISVAN. Outros, também primários, mas de maior periodicidade (consumo aliCadernos de Saúde Pública, R.J., 2 (3): 349-358, jul/set, 1986. 
mentar, produção agrícola, dados escolares, estimativas de safras) obececem a uma sistemática própria, enquadrando-se na rotina dos respectivos serviços.

As informações de fontes secundárias, como variáveis demográficas e sócioeconômicas, chegam ao SISVAN através das publicações usuais que veiculam tais informes (boletins, revistas, periódicos, livros, relatórios).

Os dados primários coletados pelo SISVAN são transcritos, criticados, digitados, processados e armazenados em computadores da Fundação Joaquim Nabuco.

\section{PROCESSAMENTO}

Como já foi referido no item anterior, os dados primários são processados em computador na Unidade Central do SISVAN, em Recife. No Estado da Paraíba, o sistema ainda não dispoe dos recursos de processamento eletronico e, assim, a nível central, as informações são tabuladas manualmente.

\section{ANÁLISE DAS INFORMAÇOES}

As informaçoes processadas pelo SISVAN, bem como os dados complementares oriundos de fontes secundárias são interpretados pelos componentes do comite multi-institucional e setor de programação com o objetivo de avaliar a tendência da situação nutricional, considerar os fatores condicionantes e aperfeiçoar a metodologia do sistema e indicar medidas para os formuladores de políticas e gestores de programas de alimentação e nutrição.

\section{DIFUSÃO}

Depois de processadas e analisadas as informaçбes sđo enviadas as unidades de prestação de serviços que compoem o SISVAN, bem como as instituições de nível regional que formam o "staff" consultivo e o quadro de coordenação/ execução do sistema.

\section{USO E USUÁRIOS DA INFORMAÇÃO}

Consideram-se, em princípio, como usuários do SISVAN todos os serviços e instituiçoes que participam de sua estru. tura e operacionalização; unidades de saúde do Estado, do INAMPS (Previdência Social), escolar, serviços de assistência técnica e extensđo agrícola, bem como as instâncias superiores de decisóes.

$\mathrm{Na}$ realidade, o uso das informações não tem sido tão extenso e efetivo como se deseja. A própria coleta de infor- 
mações e seu processamento não alcançaram o grau de diversificação e de cobertura desejado, as unidades prestadoras de serviços não assumiram os propósitos e o alcance do SISVAN. É discutivel se as instituições de formulação e coordenação de políticas de alimentação e nutrição seriam positivamente influenciadas em suas decisões.

Atualmente, se considera a conveniência de simplificar a coleta de informaçбes, centrando o sistema no setor saúde e de modo particular, nas açб̃es básicas de saúde, bem como na área de abastecimento alimentar.

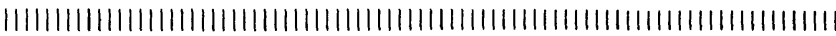

The present study descrives two projects of Food and Nutrition Surveillance System developed in the city of Recife (Pernambuco) and in the Vale do Piancó (Paraiba), Northeast Brazil. Especifically, present the institutional and operative organization, colletion of data, processing, analyse and the use given to the information produced by the system. These projects are still in experimental phase, therefore can not be talken as a definitive model. The preliminary results do not allow in this moment a consistent analyse. The National Food and Nutrition Institut (INAN, Brazil) located at central level, is re-defining the System based upon new concepts and operational orientation.

\section{REFERÊNCIAS BIBLIOGRĀFICAS}

1. ARRUDA, B.K.G. Padrões e hábitos alimentares da população brasileira. Brasilia, INAN, 1981. mimeo.

2. ARRUDA, B.K.G. A politica alimentar e nutricional do Brasil. Brasília, 1984. mimeo.

3. BATISTA FILHO, M. Saúde e nutrição. In: ROUQUAYROL, Z. Epidemiologia e saúde. 2. ed. Rio de Janeiro, MEDSI, 1986.

4. BATISTA FILHO, M. \& BARBOSA, N.P. Alimentação e nutrição no Brasil. Pró-Memória: 1974-1984. Brasîlia, Canadá Graf. Ed., 1985.

5. BECKER, R.A. et alii. Anos potenciais de vidas perdidos, Brasil, 1980. Brasilia, Divisão Nacional de Epidemiologia, 1984.

6. CALSING, C.F. et alii. Desigualdades sociais do nordeste. Brasilia, CNRH/IPEA/UNICEF, 1985. (Série instrumentos para a ação).

7. ECONOMIA e Negócios. Os números sombrios. Veja, out., 1984.

8. FUNDAÇÃO INSTITUTO BRASILEIRO DE GEOGRAFIA E ESTATISTICA. Pesquisa nacional de amostras por domicilio. PNAD, 1980. Rio de Janeiro, 1981. 
9. GOMEZ, F. Desnutrición Bol. Med. Hosp. Infant., Mexico, $3(4): 543-51,1946$.

10. LUCENA, M.A.F. et alii. Padróes alimentares de famlias rurais do trópico semi-drido (nordeste do Brasil) Alim. Nutr., 3/4 $51-8,1984$.

11. MEDEIROS NETO, G. Bócio endêmico. Levantamento de sua prevalência por micro-regiões homogêneas. $R$. bras. Malariol. D. Trop., (14) 1978.

12. NÓBREGA, F.J. (ed.) Desnutrição intra-uterina e pós-natal. São Paulo, Panamed, 1982.

13. SALOMON, J.B. \& Dర̄REA, L.J.G. Perfil da alimentação brasileira. Relação entre calorias e proteínas. R. ABIA/SAPRO, 33:14-30, 1977.

14. SANTOS, L. et alii. Xerophtalmia in the State of Paraíba, Northeast Brasil. Amer. J. Clin. Nutr., 38:139-44, 1982.

15. SHRIMPTON, R. Ecologia da desnutrição na infancia. Brasília, UNICEF, 1985. (Série Instrumento para a Ação, 3).

16. TORRES, M.A.A. Estado nutricional e condiçós sócio-econô. micas de familias rurais do trópico semi-árido (nordeste do Brasil) Recife, 1982. [Tese, Departamento de Nutrição]. 